Nota Técnica

\title{
Notas sobre la influencia agroforestal en la estabilización de parcelas y agricultores en Loreto, Perú
}

\author{
[Notes about the agroforestry influence on the stabilization of plots and \\ farmers in Loreto, Peru]
}

\author{
Gustavo Fernando Gamarra Ramírez¹, Julio Abel Soplín Ríos²
}

\begin{abstract}
1. Universidad Científica del Perú (UCP). Facultad de Ciencias e Ingeniería. Av. Quiñones km 2,5, San Juan Bautista, Maynas, Loreto, Perú. Correo electrónico: ggamarrar@hotmail.com (G. F. Gamarra* Autor para correspondencia). 2. Universidad Nacional de la Amazonía Peruana (UNAP). Facultad de Agronomía. Calle Samanez Ocampo 185, Iquitos, Perú. Correo electrónico: juasori@yahoo.es (J. A. Soplín).
\end{abstract}

\section{Resumen}

Una de las causas de la deforestación en la Amazonía peruana es la agricultura migratoria por parte de la población de las comunidades amazónicas, por lo cual tuvimos como objetivo determinar el efecto de la capacitación en sistemas agroforestales en la estabilización en el tiempo de permanencia y rotación de las parcelas, del agricultor en la comunidad de San Regis, río Marañón (Loreto, Perú). La muestra fueron 32 agricultores con un promedio de 2 hectáreas de cultivos en producción. Se aplicó pre-test y pos-test. Los resultados muestran que la capacitación y la asistencia técnica sobre los sistemas agroforestales influyen en la permanencia en la comunidad de 5,1 a 7,5 años (75\%); y referente a la rotación de los sistemas de producción de los cultivos se obtuvo, que en el pre-test que 29 agricultores $(90,6 \%)$ practicaban el monocultivo como sistema de siembra y, en el pos-test, 25 agricultores $(78,1 \%)$ practican agroforestería. Concluyendo que la capacitación y asistencia técnica para los productores de las comunidades ribereñas, contribuye en la conducción de sus agrosistemas y el uso eficiente del suelo.

Palabras clave: Amazonía peruana, Capacitación, Cultivos, San Regis, Sistemas agroforestales.

\begin{abstract}
One of the reasons of deforestation in the Peruvian Amazonia is the migratory agriculture carried out by the population of amazonian communities. In this respect, our aim was to determine how the training on agroforestry systems affects the permanence and rotation of the plots belonging to the population of the San Regis community, Marañon River (Loreto, Peru). The samples were 32 farmers each with about two crop hectares, which were in production at the moment of the study. Pre- and post-tests were applied. The results show that training and technical assistance on agroforestry systems have an influence on the permanence of the community between 5,1 to 7,5 years (75\%). Regarding to the rotation of crop productive systems, it was observed that in pre-test, 29 farmers $(90,6 \%)$ had monoculture as sowing systems, and that in post-test, 25 farmers $(78,1 \%)$ practiced agroforestry. We conclude that the training and the technical assistance given to the riverside communities contribute to the handling of their agrosystems and to the efficient use of the soils.
\end{abstract}

Keywords: Agroforestry systems, Crops, Peruvian Amazon, San Regis, Training. 


\section{INTRODUCCIÓN}

La agricultura tradicional en el trópico, se basa en la tala y quema de áreas boscosas para reemplazarla por cultivos (Vallejo et al., 2011; Rojas y Fabre, 2017); se trata de una dinámica donde el agricultor va substituyendo un ecosistema natural por un ecosistema agrícola con mucho menos diversidad que el sistema original. La agricultura migratoria comprende sistemas de subsistencia orientadas a satisfacer las necesidades básicas de alimentos (Ravikumar et al., 2017). Sólo ocasionalmente considera la fuente de ingresos por la venta excedente de su producción.

En este sistema el bosque se corta, junta y quema y la tierra se cultiva en pocos años, luego del periodo de cultivo continúa una fase de descanso. Inicialmente, la productividad del cultivo es elevado ya que con la quema los nutrientes se incorporan al suelo, haciéndolos más fértiles. Pero después de 4 años los suelos se empobrecen debido a la siembra de monocultivos y exigencia de nutrientes de los mismos; para luego quedar en descanso (barbechos) por espacios de años prolongados, esperando la recuperación del suelo por regeneración natural (Brack, 1994).

Esta agricultura migratoria los conlleva a una degradación acelerada de sus suelos y una migración continua de los pobladores hacia nuevas áreas boscosas, repitiéndose el círculo vicioso. Sin embargo, se ha podido comprobar que existen experiencias muy interesantes y eficientes de sistemas agroforestales, que no son difundidas y en forma adecuada entre los pobladores rurales (Kalliola y Flores, 1998).

La agroforestería como ciencia es reciente, fue institucionalizada en 1967 con la creación del Consejo Internacional para la Investigación Agroforestal (ICRAF) con sede en Nairobi, Kenya. En el ambiente amazónico, al igual que en otros ambientes tropicales del mundo, la agroforestería es una denominación nueva para una práctica milenaria de la población aborigen, que puede ofrecer para el desarrollo moderno alternativas realistas para utilizar las mismas tierras en el largo plazo. Ecológicamente, conservando los recursos naturales, económicamente por diversificación de la producción con rentabilidad, y socialmente estabilizando a la familia campesina (Kalliola y Flores, 1998). Los sistemas agroforestales constituyen modelos de producción sostenible y eficiente para la utilización de la tierra, con el propósito fundamental de diversificar y optimizar la producción, al mismo tiempo de respetar los principios básicos de la sostenibilidad (JIRCAS, 2010).

La agricultura migratoria no es ajena a los agricultores de la localidad de San Regis, río Marañón, distrito de Nauta, quienes por muchos años han intervenido el bosque con monocultivos de ciclo corto ocasionando la pérdida de la capacidad productiva de los suelos y abandono a la regeneración natural de la vegetación sucesional. El manejo de la vegetación natural sucesional de la purma constituye la alternativa a la deforestación y al mal uso de la tierra (Dourojeanni, 2013).

El sistema agroforestal en las parcelas de los agricultores de la comunidad de San Regis contribuirá a mejorar sus condiciones de vida ya que le garantizará mayor seguridad en la producción de las parcelas por ofrecer diversidad de productos, tanto para el autoconsumo (alimentos, leña, fibras, medicinas, etc.) como para los mercados cercanos (frutas, carne de animales silvestres, hojas, etc.). Constituyen, en muchos casos, una forma de ahorro y capitalización para los productos rurales.

Por lo cual el objetivo fue determinar el efecto de la capacitación en sistemas agroforestales en la estabilización en el tiempo de permanencia y rotación de las parcelas, del agricultor en la comunidad de San Regis, río Marañón (Loreto, Perú). 


\section{MATERIALES Y MÉTODO}

\section{Área de estudio}

Las encuestas se realizó en la comunidad de San Regis, ubicada en la cuenca del río Marañón, distrito Nauta, provincia Loreto, Perú (Figura 1); en las coordenadas geográficas UTM: 622420, 9501760, Zona 18. En cuanto al suelo donde se desarrollan los sistemas agroforestales en la comunidad son franco arenoso y franco arcilloso limoso, con pH extremadamente ácido $(<4,5)$, fósforo disponible de medio a bajo, potasio variado (entre bajo, medio y alto), materia orgánica medio; por lo tanto, fertilidad natural media. El tipo de vegetación corresponde a los cultivos de varias especies y bosques de terrazas medias (Zárate et al., 2013). La fisiografía corresponde a: colinas bajas moderadamente disectadas, y terrazas medias, con pendientes de 2 a $4 \%$ (Escobedo y Torres, 2012); en cuanto al clima, la precipitación fluctúa en las ciudades de Iquitos y Nauta de $2500-2700 \mathrm{~mm} / \mathrm{año}$, con promedios de temperatura mínimas del aire de $20-23^{\circ} \mathrm{C}$ y máximas de $30-33^{\circ} \mathrm{C}$. Y las medias anuales están en $27^{\circ} \mathrm{C}$ (Paredes, 2012). La fisiografía corresponde a: colinas bajas moderadamente disectadas, y terrazas medias, con pendientes de 2 a 4\% (Escobedo y Torres, 2012).

\section{Técnica de recolección de datos}

Para la recopilación de información se elaboró una encuesta de 29 preguntas al grupo experimental con un pre-test y luego un pos-test. Estas encuestas cuentan con peguntas cerradas y abiertas, referente a las dimensiones de aspectos generales, aspectos sociales, aspectos productivos, aspectos técnicos de los sistemas agroforestales y la estabilización en sus parcelas. Las preguntas realizadas se detallan en el Anexo 1.

\section{Análisis de datos}

La población de estudio estuvo conformada por todos los agricultores de la comunidad de San Regis (216 agricultores) y se tomó como muestra a 32 agricultores, con un promedio de edad entre 35 a 50 años de ambos sexos, con un promedio de 2 ha de cultivos en producción.
Para medir el efecto de la capacitación en los sistemas agroforestales en la estabilización en el tiempo de permanencia y rotación de las parcelas del agricultor en la comunidad de San Regis se utilizó la prueba de McNemar-Bowker y chi-cuadrado, ya que deseábamos conocer si existe diferencia entre el antes (pre-test) y el después (pos-test) de la capacitación sobre los sistemas agroforestales a los agricultores de la comunidad de San Regis. Esta prueba de McNemar-Bowker, se utiliza en variables cualitativas apareadas, en este caso son apareadas porque es la misma población en la que midió la misma variable antes y después ( $3 \times 3)$; y este apareamiento es para demostrar si la capacitación tuvo un efecto sobre las diferentes variables estudiadas. La confiabilidad del instrumento para la recolección de datos (encuesta, pre-test y pos-test) fue determinado utilizando el coeficiente de alpha de Cronbach; esta herramienta permite medir la precisión de la prueba a aplicar y la ausencia de errores en un test (Hernández et al., 2002).

\section{RESULTADOS}

Al realizar la prueba piloto de confiabilidad del pre-test y pos-test, se obtuvo el valor de 0,624 (alpha de Cronbach), categorizada como buena, reportando que el instrumento utilizado, es decir, el test aplicado a los agricultores, que formaron el grupo experimental (al inicio y al final), expresa que es confiable estadísticamente al $62,4 \%$. El tiempo de permanencia de muchos agricultores en su comunidad fue de 0,0 a 2,5 años para el $75 \%$ y de 2,6 a 5,0 años para el $21,9 \%$ (ver mayores detalles en la Tabla 1). Por otro lado en el pos-test aplicado después de la capacitación se encontró a 2 agricultores $(6,3 \%)$ con permanencia de 0,0 a 2,5 años en su comunidad, así mismo 6 agricultores $(18,7 \%)$ con permanencia de 2,6 a 5 años; y 24 agricultores (75\%), es decir el mayor número de encuestados tienen permanencia en la comunidad de 5,1 a 7,5 años.

Esto se confirma con la prueba de McNemar-Bowker (Tabla 2), que utilizando la prue- 


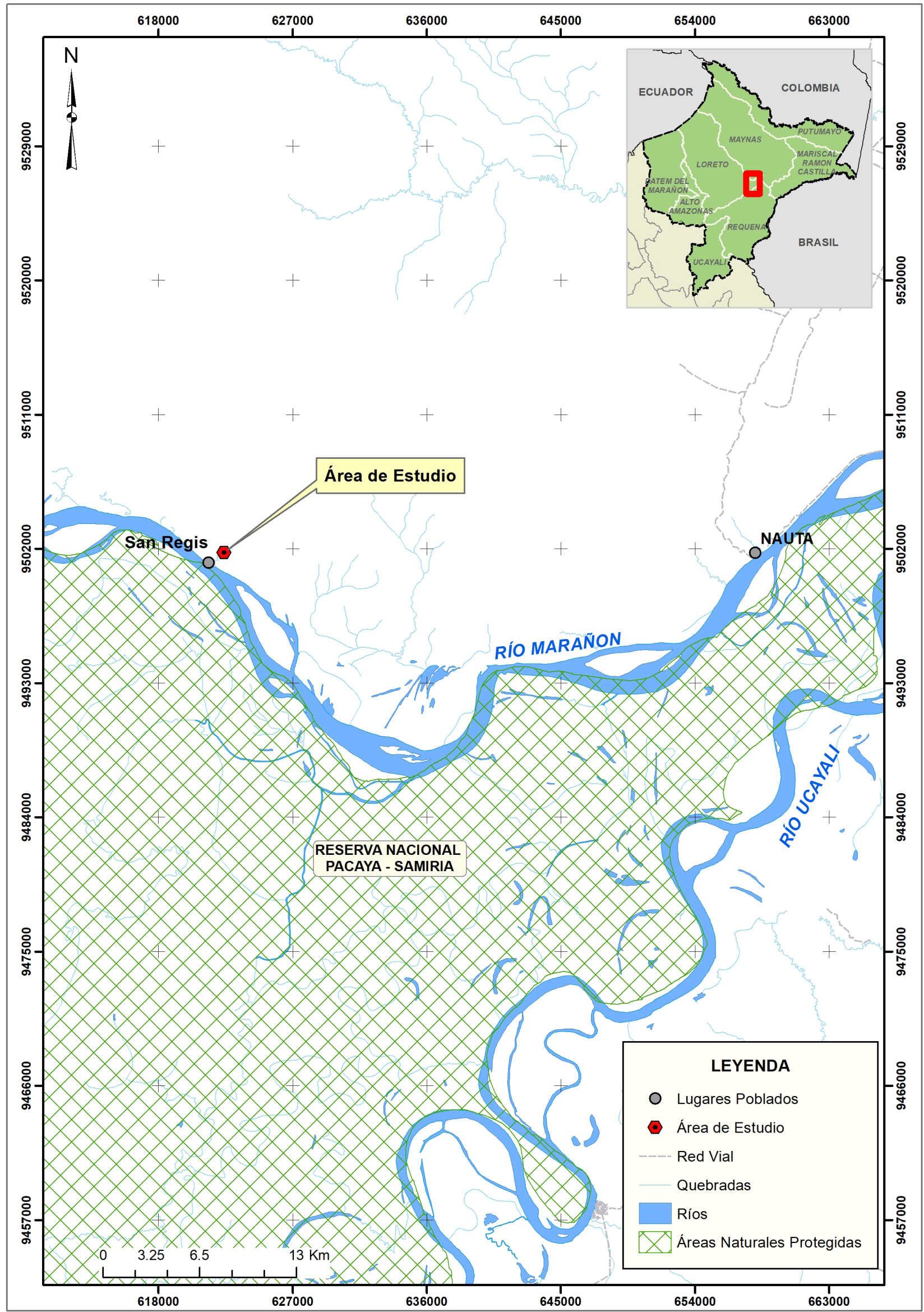

Figura 1. Ubicación de la comunidad de San Regis, rio Marañón, Amazonía peruana; donde se realizó el estudio. 
ba de contraste de chi-cuadrado, del pre-test y pos-test del tiempo de permanencia del agricultor en la comunidad, nos indican que estos promedios son estadísticamente diferentes.

La Tabla 3 muestra si la existencia de las parcelas agroforestales determinó su permanencia en la comunidad, en referencia al pre-test aplicado, se encontró que en 6 agricultores (18,8\%) las parcelas agroforestales eran determinante para su permanencia en su comunidad; así mismo en 5 agricultores $(15,6 \%)$ se observó que las parcelas agroforestales no influían casi nada para su permanencia en su comunidad; y en 21 agricultores $(65,6 \%)$ se estableció que las parcelas agroforestales no eran determinantes para su permanencia en su comunidad.

En cambio, en referencia al pos-test aplicado, se encontró que en 23 agricultores (71,9\%) las parcelas agroforestales eran determinantes para su permanencia en su comunidad; así en 8 agricultores $(25,0 \%)$ se observó que las parcelas agroforestales no influían casi nada para su permanencia en su comunidad; y en 1 agricultor $(3,1 \%)$ se estableció que las parcelas agroforestales no eran determinantes para su permanencia en su comunidad.

Esto se confirma en la Tabla 4, con la prueba de McNemar-Bowker, que utilizando la prueba de contraste de chi-cuadrado, del pre-test y postest, que la existencia de las parcelas agroforestales determinó su permanencia en la comunidad, son estadísticamente diferentes.

\section{DISCUSIÓN}

Se establece que la capacitación mediante los curso-talleres de sistemas agroforestales ha tenido una influencia determinante en la permanencia de los pequeños agricultores en la comunidad, además estas capacitaciones prácticas y, la implementación de las parcelas agroforestales demostrativas $(71,9 \%)$ ha sido determinante en esta decisión. Esto lo corrobora Pérez (2014); quien encontró que el 71,4\% de los productores ribereños permanecen viviendo más de 21 años en las comunidades donde rea- lizó su estudio; este porcentaje, demuestra que ellos son poco nómadas (migración permanente del lugar), por el contrario, son más sedentarios por la capacitación y la implementación de sistemas agroforestales y diversifican su producción agrícola, ya que el uso de la tierra es más frecuente, logrando desarrollarse agricultura más intensa. Además, White et al. (2005) encontraron una relación directa de la productividad de los agrosistemas con el bienestar de la población, es decir, a pesar de obtener rendimientos aceptables de algunos cultivos no se generan cambios en los aspectos sociales y económicos en los hogares, estos pobladores continúan viviendo en la pobreza y extrema pobreza; se encuentran desmotivados por los bajos precios de sus productos y lo comercializan al mejor postor. Este hecho viene ocasionando la postergación del anhelo de mejorar el nivel de vida de nuestros pobladores ribereños amazónicos. También se señala que un elemento que caracteriza a los hogares rurales en los últimos años, además de la diversificación de sus actividades económicas, es también la migración hacia otras zonas con el objetivo de conseguir mayores ingresos (Yamada, 2012). Asimismo, Pérez (2014), referente al ingreso económico mensual del agricultor; observó en el pre-test que el $88,6 \%$ tuvieron ingresos entre $\mathrm{S} / 150$ a S/750 soles y luego en el pos-test se mostró que el 54,3\% tuvieron ingresos desde S/751 soles a más; continúa señalando que existe una relación directa de los ingresos económicos con los agrosistemas; es decir que una mayor diversificación de la producción, genera mayores ingresos por la venta de los productos. Además, que estos ingresos les permite optar por otras actividades generadores de recursos como son la pesca, el comercio, extracción de madera, servicio de transporte fluvial, etc. $Y$ en términos generales se mejora a condición tanto económica como social del poblador ribereño. Aunque los precios que reciben por su producción son muy bajos.

Así mismo, como lo muestra el resultado de la determinación en el tiempo a quedarse en ese lugar manejando sus Sistemas agroforestales (más de 4 años), existen 22 agricultores 
Tabla 1. Recuentro (y porcentaje en paréntesis) de agricultores del tiempo de permanencia en la comunidad de San Regis antes y después de la capacitación $(n=32)$.

\begin{tabular}{ccc}
\hline Clase & Pre-test & Pos-test \\
\hline 0 a 2,5 años & $24(75,0 \%)$ & $2(6,3 \%)$ \\
2,6 a 5 años & $7(21,9 \%)$ & $6(18,8 \%)$ \\
5,1 a 7,5 años & $1(3,1 \%)$ & $24(75,0 \%)$ \\
\hline
\end{tabular}

Tabla 2. Prueba de McNemar-Bowker para el tiempo de permanencia del agricultor en la comunidad.

\begin{tabular}{cccc}
\hline & Valor & Gl & p-valor (bilateral) \\
\hline Prueba de McNemar-Bowker & 24,000 & 2 &, 000 \\
N de casos válidos & 32 & & \\
\hline
\end{tabular}

Tabla 3. Recuentro (y porcentaje en paréntesis) de para observar si la existencia de las parcelas agroforestales determinó su permanencia en la comunidad $(n=32)$.

\begin{tabular}{ccc}
\hline Clase & Pre-test & Pos-test \\
\hline Es determinante & $6(18,8 \%)$ & $23(71,9 \%)$ \\
Casi ni influye & $5(15,6 \%)$ & $8(25,0 \%)$ \\
No es determinante & $21(65,6 \%)$ & $1(3,1 \%)$ \\
\hline
\end{tabular}

Tabla 4. Prueba de McNemar-Bowker para observar si la existencia de las parcelas agroforestales determinó su permanencia en la comunidad.

\begin{tabular}{ccccc}
\hline & Valor & GI & Significación asintótica (bilateral) \\
\hline Prueba de McNemar-Bowker & 20143 & 3 &, 000 \\
N de casos válidos & 32 & & \\
\hline
\end{tabular}

(68,8\%) que continúan trabajando en sus parcelas con sistemas agroforestales actualmente. La capacitación en sistemas agroforestales nos demuestra que 25 agricultores (78,10\%) cuentan con sistemas agroforestales como sistema de siembra; en relación a 29 agricultores $(90,80 \%)$ que manejaban monocultivos anteriormente, es decir antes de la capacitación (pre-test). Estas capacitaciones han permitido que 23 agricultores (71,90\%) y 7 agricultores $(21,90 \%)$, manejen más de 2 ha, y hasta 2 ha, con sistemas agroforestales respectivamente.
Todo esto como resultado del pre-test aplicado a los agricultores que los ha motivado a establecerse en sus parcelas bajo el sistema agroforestal.

La metodología empleada en la capacitación de los sistemas agroforestales de producción, fueron a partir de las necesidades sentidas en los agricultores, es decir, desde la fase de planificación; la ejecución de los cursos talleres y la instalación de los sistemas agroforestales, así como de resultados concretos en el cam- 
po; estos aspectos han sido considerados por los agricultores de la comunidad de San Regis como la que ha contribuido a resolver los problemas técnicos en la producción. Es así que se tiene que 23 de ellos consideran que sí resolvió su problema agrícola; y 8 agricultores lo consideran de forma regular que apoyo en sus problemas agrícolas. A través de los eventos de capacitación dirigida a los 32 agricultores de la comunidad de San Regis, se ha notado cambios en la actitud, pues durante este proceso de los cursos talleres han participado activamente, desde la fase de planificación, ejecución y resultados. La promoción e introducción de tecnologías apropiadas que valoren e integren a la cultura y costumbres de la comunidad (proceso de enseñanza-aprendizaje) ha fortalecido las capacidades y mejora en sus actividades productivas de los agricultores mediante los sistemas agroforestales de producción.

En lo relacionado a la mejora de las técnicas que aplican en sus agrosistemas del agricultor, Pérez (2014); observó que en el pre-test el 14,3\% manifiesta que si mejoró su técnica y; luego en el pos-test el $51,4 \%$ de los agricultores señalan que su técnica agrícola si mejoro. Esta versión se refuerza porque, paralelamente, la investigación y extensión agrícola avanzo poco, y no ha logrado desarrollarse tanto por el lado de la oferta, como por el lado de la demanda, pero se nota algunos avances en la adopción de técnicas agrícolas para incrementar la producción de pan llevar en los productores.

Referente al grado de instrucción del agricultor, Trivelli (2000) encontró que el 62,8 \% tienen hasta primaria incompleta; mientras que el $11,4 \%$ estudiaron hasta completar la secundaria; continúa señalando que los hogares con mayores ingresos son aquellos que cuentan con más educación formal y con familias de menor tamaño. Cabe resaltar, sin embargo, que los niveles educativos de los jefes de hogares rurales son más bien bajos, ya que alcanzan en promedio sólo a primaria completa. Además, concerniente al estudio (MINAG, 2010), revela que el bajo nivel educativo de la población rural es una seria limitación para el desarrollo de capacidades de los agricultores: sólo el 34\% de los agricultores tiene primaria completa, el 14\% secundaria completa, y el $4 \%$ tiene estudios superiores. Estos hechos limitan la capacidad de los productores para la innovación tecnológica y para su capacidad de gestión. Igualmente, (INEI- ENAHO, 2005 -2010) demuestra que en el Perú; señala 3 de cada 4 agricultores son pobres y se caracterizan por tener bajos niveles de conocimientos tecnológicos para la producción. Es importante indicar que los centros e instituciones amazónicas, dedicadas a la extensión y promoción agropecuarias, deberían poner un alto esfuerzo en recoger las experiencias de los pobladores y difundirlas. Esto también ayudaría a valorar las experiencias de los pobladores, porque muchos de los sistemas han sido desarrollados o son practicados por ellos.

Se puede concluir que el diseñar un programa de capacitación y asistencia técnica para los productores de las comunidades ribereñas, contribuye en la conducción de sus agrosistemas y el uso eficiente del suelo mediante la asociación adecuada de cultivos, además de mejorar el nivel de adopción de las tecnologías generadas por las instituciones de investigación.

\section{AGRADECIMIENTO}

Sincero agradecimiento a los pobladores y autoridades de la comunidad de San Regis por permitirnos desarrollar la presente investigación en sus localidad.

\section{REFERENCIAS BIBLIOGRÁFICAS}

Brack, E A. (1994) Sistemas agroforestales e importancia de la agroforestería en el desarrollo de la selva central. San Ramón: INFOR-GTZ.

Centro Internacional de Investigación de las Ciencias Agropecuarias del Japón - JIRCAS(2010) Manual de Sistemas Agroforestales para el Desarrollo Rural Sostenible. Febrero, 2010. San Lorenzo. 
Dourojeanni, M. J. (2013). Loreto sostenible al 2021. Loreto: DAR, Derecho, Ambiente y Recursos Naturales.

Escobedo, R. y Torres, G. (2012) Suelos y capacidad de uso mayor de las tierras, documento temático. Proyecto Microzonificación Ecológica y Económica del Área de Influencia de la Carretera Iquitos-Nauta. Iquitos: IIAP y DEVIDA.

Escobedo, R. y Torres G. (2012) Fisiografía, documento temático. Proyecto Microzonificación Ecológica y Económica del Área de Influencia de la Carretera Iquitos-Nauta. Iquitos: IIAP y DEVIDA.

Hernández, S.R; Fernández, C.C y Baptista, L.P. (2002) Metodología de la investigación. Vol. 4. Ed. A cargo de Mcgraw-Hill / Interamericana Editores, S.A. México: McGraw-Hill Interamericana.

Kalliola, R. y Flores Paitán, S. (1998) GEOECOLOGÍA Y DESARROLLO AMAZÓNICO: EStUdio integrado en la zona de Iquitos, Perú. Finlandia: Turun Yliopisto

MINAG. (2010) Estadísticas agropecuarias del Perú. Ministerio de Agricultura. Oficina Sectorial de Estadísticas Agrarias. MINAG. Lima: MINAG.

Paredes, M. (2012) Clima, documento temático. Proyecto Microzonificación Ecológica y Económica del Área de Influencia de la Carretera Iquitos-Nauta. Ed. A cargo del IIAP y DEVIDA. Iquitos: IIAP y DEVIDA

Pérez, A, J. E. (2014) Agrosistemas de suelos aluviales en los niveles socioeconómicos del poblador ribereño de la provincia de Maynas. Loreto-Perú. Iquitos: Escuela de Posgrado de la Univrsidad nacional de la Amazonía Peruana.

Ravikumar, A., Sears, R. R., Cronkleton, P., Menton, M., y Pérez-Ojeda del Arco, M. (2017). Is small-scale agriculture really the main driver of deforestation in the Peruvian Amazon? Moving beyond the prevailing narrative. Conservation Letters, 10(2), 170-177.

Rojas, G. G., y Fabre, N. (2017). Agroecologia e mudanças climáticas no Trópico Semiárido. REDES: Revista do Desenvolvimento Regional, 22(2), 174-188.

Trivelli, C. (2000) Pobreza rural: investigaciones, mediciones y políticas públicas en: Perú: El problema agrario en debate. Sepia VIII. Ed. por Hurtado, I; C. Trivelli, A. Brack. Lima: SEPIAIRD- ITDG, pp 199-255.

Vallejo Nieto, M. I., Gurri García, F. D., y Molina Rosales, D. O. (2011). Agricultura comercial, tradicional y vulnerabilidad en campesinos. Política y cultura, 36, 71-98.

White, D.; Labarta, R. y Leguia, E. (2005) Technology adoption by resource-poor farmers: considering the opportunity cost of peak-season labor. Agricultural Systems, 85(2):183-201.

Yamada, G. (2012). Patrones de migración interna en el Perú reciente. Empleo y protección social, 91-124.

Zárate, R.; Mori, T. y Maco, J. 2013. Estructura y Composición Florística de las Comunidades Vegetales del ámbito de la Carretera Iquitos-Nauta, Loreto, Perú. Folia Amazónica, 22 (1-2): 77-89.

\section{Conflicto de interés}

Los autores indicamos que no tenemos conflictos de intereses en ningunas de las fases de investigación y publicación del presente trabajo. Decidimos que el autor para correspondencia sea G. F. Gamarra ya que este trabajo representa su tesis de postgrado en la universidad. 
Anexo 1. Instrumento de recolección de datos (pre-test y pos-test) en la localidad de San Regis.

\section{Información general}

1.1 Fecha de aplicación:

1.2 Tiempo de permanencia en la comunidad:
1). $0-2,5$ años ( )
2). 2,6-5 años ( )
3). 5,1-7,5 años ( )

\section{Visita a parcelas}

2.1. ¿Qué sistema de producción utilizaba en años anteriores?
1). Monocultivo ( )
2). Agroforestería ( )
3). Otros ( )

2.2. ¿Qué áreas cultivaba en cada sistema?
1). Monocultivo: $0,5-2,00$ ha
2). Agroforestería: $0,2-0,5$ ha
3). Otros: $1,00-2,00$ ha

2.3. En términos porcentuales, cual es el destino de la producción:
1). Autoconsumo ( )
2). Autoconsumo/venta ( )
3). Venta ( )

2.4. Por los antecedentes en rendimiento de producción y económico del cultivo, que decidió usted.
1). Continuar con mis monocultivos ( )
2). Cambiar por agroforestería
3). Aun no lo he decidido ( )

2.5. ¿Qué sistemas de cultivos practica actualmente?
1) Monocultivo( )
2) Agroforestería ( )
3) $\operatorname{Otros}()$

2.6. ¿Con que áreas de monocultivo cuenta?
1) De 0,5 a 1,0 ha ( )
2) de 1,1 a 2,0 ha ( )
3) de 2,1 a 3,0 ha ( )

2.7. ¿Con que áreas de agroforestería cuenta?
1) Aun no instale ( )
2) De 0,5 a 1,0 ha ( )
3) de 1,1 a 2,0 ha ( )

III. Establecimiento en sus parcelas Agroforestales y tiempo de permanencia en el lugar

3.1 ¿Cuántos años tiene establecido sus parcelas agroforestales?
1) 1 año ( )
2) 2 años ( )
3) Más de 3 años ( )

3.2 ¿Cuantas Hectáreas viene manejando su parcela con sistema agroforestal?
1) De 0,5 a 1,0 ha ( )
2) de 1,1 a 2,0 ha ( )
3) más de 2,0 ha ( )

3.3 ¿La existencia de sus parcelas agroforestales determinan su permanencia en el lugar por mayor tiempo?
1) Es determinante ( )
2) casi no influye ( )
3) no es determinante ( )

3.4. ¿Cuánto tiempo más estimas quedarte en este lugar manejando tus parcelas agroforestales?
1) De 1 a 2 años ( )
2) de 2 a 4 años ( )
3) Por más de 4 años ( )

3.5. ¿Está usted satisfecho viviendo en esta comunidad?
1) Satisfecho ( )
2) No satisfecho ( )
3) medianamente satisfecho ( )

\section{Asistencia técnica y apoyo recibido}

4.1 La institución que te apoyo para la instalación de los sistemas agroforestales fue:
1) Publica ( )
2) Privada ( )
3) Ambas ( )

4.2 ¿La asistencia técnica recibida resolvió tus problemas agrícolas?
1) $\mathrm{Si}($ )
2) No ( )
3) En forma regular ( ) 